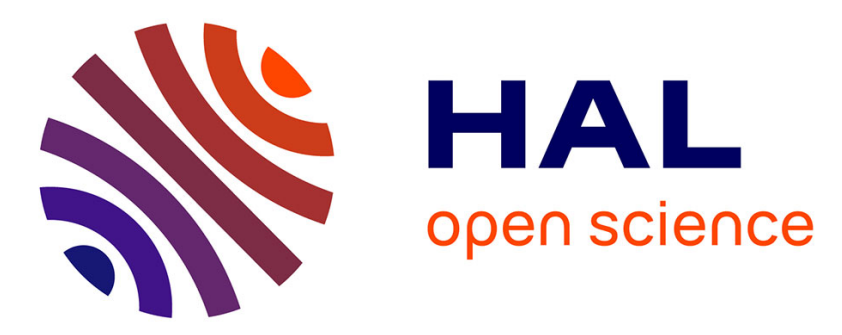

\title{
Remaining useful life estimation based on nonlinear feature reduction and support vector regression
}

T Benkedjouh, Kamal Medjaher, Noureddine Zerhouni, S Rechak

\section{To cite this version:}

T Benkedjouh, Kamal Medjaher, Noureddine Zerhouni, S Rechak. Remaining useful life estimation based on nonlinear feature reduction and support vector regression. Engineering Applications of Artificial Intelligence, 2013, 26 (7), pp.1751 - 1760. hal-03223494

\section{HAL Id: hal-03223494 \\ https://hal.science/hal-03223494}

Submitted on 11 May 2021

HAL is a multi-disciplinary open access archive for the deposit and dissemination of scientific research documents, whether they are published or not. The documents may come from teaching and research institutions in France or abroad, or from public or private research centers.
L'archive ouverte pluridisciplinaire HAL, est destinée au dépôt et à la diffusion de documents scientifiques de niveau recherche, publiés ou non, émanant des établissements d'enseignement et de recherche français ou étrangers, des laboratoires publics ou privés. 


\title{
Remaining useful life estimation based on nonlinear feature reduction and support vector regression
}

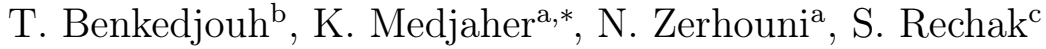 \\ ${ }^{a}$ FEMTO-ST Institute, UMR CNRS 6174 - UFC / ENSMM / UTBM \\ Automatic Control and Micro-Mechatronic Systems Department \\ 24, rue Alain Savary, 25000 Besançon, France \\ ${ }^{b}$ EMP, Laboratoire de Mécanique des Structures (LMS), Bordj El Bahri, Algiers, Algeria \\ ${ }^{c}$ ENSP, Laboratoire de Génie Mécanique, El-Harrach, Algiers, Algeria
}

\begin{abstract}
Prognostics and Health Management (PHM) of rotating machines is gaining importance in industry and allows increasing reliability and decreasing machines' breakdowns. Bearings are one of the most components present in mechanical equipments and one of their most common failures. So, to assess machines' degradations, fault prognostics of bearings is developed in this paper. The proposed method relies on two steps (an offline step and an online step) to track the health state and predict the remaining useful life (RUL) of the bearings. The offline step is used to learn the degradation models of the bearings whereas the online step uses these models to assess the current health state of the bearings and predict their RUL. During the offline step, vibration signals acquired on the bearings are processed to extract features, which are then exploited to learn models that represent the evolution of the degradations. For this purpose, the isometric feature mapping reduction technique (ISOMAP) and support vector regression (SVR) are used.

The method is applied on a laboratory experimental degradations related to bearings. The obtained results show that the method can effectively model the evolution of the degradations and predict the RUL of the bearings.
\end{abstract}

Keywords: Prognostics and Health Management, Remaining Useful Life, Isometric Feature Mapping, Support Vector Regression

\footnotetext{
*Corresponding author. Tel.: +330381402796

Email address: kamal.medjaher@ens2m.fr (K. Medjaher)
} 


\section{Introduction}

Prognostics and Health Management (PHM) of industrial systems is a central activity of intelligent maintenances, such as Condition-Based Maintenance (CBM) and predictive maintenance (PM). PHM deals with condition monitoring, fault detection, fault diagnostics, fault prognostics and decision support. It can concern the whole industrial system as well as its critical components. The analysis of the experience feedback performed on electrical machines by the Electric Power Research Institute (ERPI), and researchers in the reliability of electrical machines, has shown that the bearings and the stator are the components which present the most failures [1]. Consequently, doing PHM on these components may increase the availability, the reliability and security of the machines. The purpose of PHM on rotating machinery is not only to detect the faults, but also to predict how much longer the machine can operate safely and perform its function. Interesting reviews on prognostics are given in $[2,3]$. Failure prognostics can be done by using three main approaches: model-based, data-driven and hybrid prognostics. among these approaches, data-driven prognostics offers a trade off in terms of precision and complexity.

Bearings' prognostics targets the prediction of RUL in order to minimize the time breakdown and maintenance costs. Most of prognostic methods related to bearings can be considered within the data-driven approach and use vibrations analysis [2]. In this framework, Shao et al. [4] proposed a progression-based prediction model for remaining useful life of bearings, [5] estimated the RUL of bearings by using both a proportional hazard model and a logistic regression. Gebraeel et al. [6] used the Feedforward Neural Networks (FFNNs) to project the degradation by computing exponential parameters that give the best exponential fit. Similarly, Huang et al. [7] proposed a self organizing map (SOM) and an artificial neural network based method for performance degradation assessment and residual life prediction of bearings, Yan et al. [8] utilized a logistic regression to achieve machine performance assessment and finally, Lingjun et al. [9] applied support vector data description (SVDD) to assess the equipment health state and to detect bolt crack.

One of the main challenges in prognostic of bearings is how to construct and evaluate health indicators from available features, which can represent the degradation states. In practice, the construction of health indicators depends on the nature of the degradations and the related monitoring data 
provided by the sensors [10]. In this domain, the raw monitoring signals are pre-processed and used to extract features. However, the number of features can be of high dimensionality and can be reduced before building the health indicator. Various techniques for data reduction have been proposed in the literature [11]. Among these techniques, Principal Component Analysis (PCA) [12] is one of the most used. Thus, Liao and Lee [13] utilized the PCA to extract features by using wavelet packet decomposition (WPD) on vibration signals of bearings. Recently, Malhi et al. [14] proposed a PCAbased feature selection approach for bearing fault classification. However, PCA is a linear reduction technique.

The main contribution of this paper concerns the utilization of the isometric feature mapping (ISOMAP) technique, to perform nonlinear feature reduction, combined with nonlinear support vector regressions (SVR) to construct health indicators allowing the estimation of the health state of bearings and predict their RUL. The purpose of the ISOMAP technique is to find a small number of features that represent a large number of observed dimensions. ISOMAP has the advantage to be nonlinear and non-iterative and gives globally optimal solutions [15]. The objective of the SVR is to estimate the relation between an input and output random variable under the assumption that the joint distribution of the input and the output variables is completely unknown. The SVR technique has been successfully applied in various machine learning problems, which are especially prominent for regression [16] and in different applications such as sunspot frequency prediction [17] and drug discovery [18]. In this paper, the SVR is used to learn the nonlinear degradation models of the bearings.

The method proposed in this paper is divided into two steps: an offline step and an online step. The offline step is used to learn the bearings' degradation models by using the ISOMAP and the SVR techniques. This step is also used to learn more about the variability of the monitoring data, to tune the parameters of the ISOMAP and SVR techniques and to define the failure thresholds of the bearings. The online step uses the models learned during the offline step to assess the current health state of new tested bearings and to predict their RUL.

This paper is organized as follows. Section 2 presents the framework for component-based PHM, section 3 describes the proposed method for RUL estimation of bearings based on ISOMAP and SVR, section 4 deals with experimental verification and results and finally, section 5 concludes the paper. 


\section{Component-oriented Prognostics and Health Management}

Prognostics and Health Management (PHM) is a central activity for the implementation of Condition Based Maintenance (CBM) and Predictive Maintenance (PM). PHM includes seven modules [19], one of them is failure prognostics. The international standard organization defines failure prognostics as the estimation of the operating time before failure and the risk of existence or later appearance of one or more failure modes [20], whereas most reported literature related to PHM defines it as the estimation of Remaining Useful Life (RUL) [2, 21, 3]. The estimation of RUL can be done by using three main approaches: model-based prognostics (also called physics of failure), data-driven prognostics and hybrid prognostics (combination of both previous approaches). Each one of these approaches has its strength and its weakness. Model-based prognostics gives more precise results, but its implementation is difficult because in most applications the construction of the physical model is not a trivial task. Data-driven prognostics relies on the data provided by the sensors to extract features which are then used to build models for RUL estimation. This approach is easy to implement but the results it provides are less precise than those of model based approach. Finally, hybrid approach takes the best of both previous approaches, but also some of their weaknesses.

The contribution presented in this paper belongs to data-driven prognos-

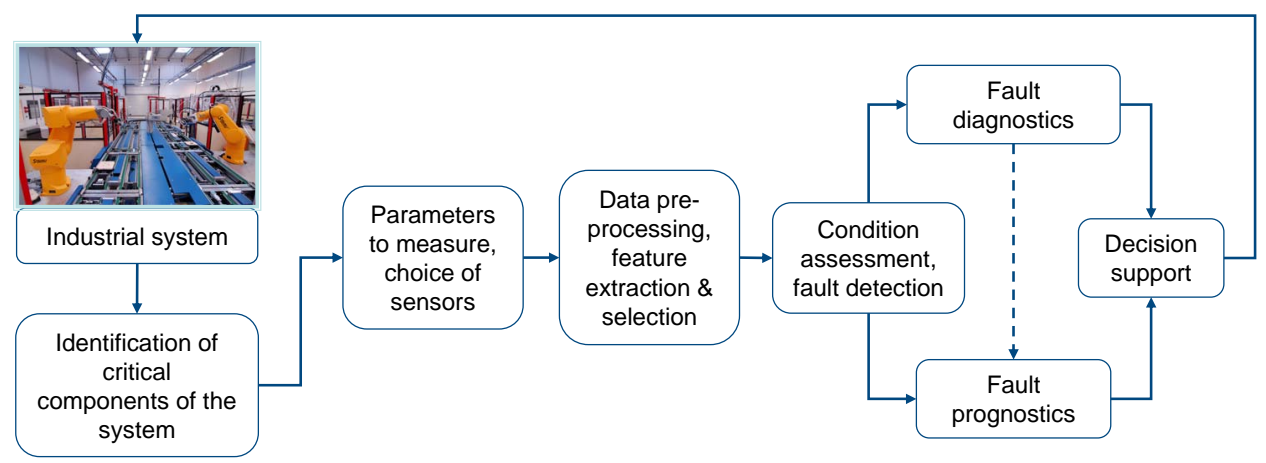

Figure 1: Component-oriented PHM framework.

tics with the hypothesis that the RUL estimation is done on the critical components of the industrial system. Figure 1 shows the framework of the component-oriented prognostics. 
In this paper we make assumption that the prognostic of the whole industrial system corresponds to prognostic of its critical components. The first step of the prognostic process is then to identify the critical components. This step is followed by the definition of the degradation phenomena, the parameters to monitor and the sensors to install. The raw data provided by the sensors are then processed to extract features and health indicators, which are used to do fault detection, diagnostics, prognostics and decision support on the industrial system. In the following of the paper, only feature extraction/reduction and fault prognostics are concerned.

\section{RUL estimation based on ISOMAP and SVR}

The main steps of the proposed contribution are shown in figure 2. Feature extraction and reduction and degradation modeling steps will be described in the following of the paper.

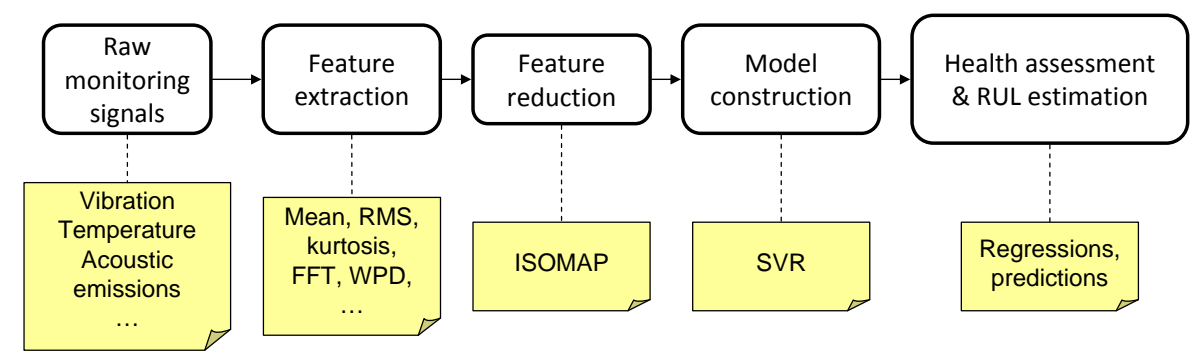

Figure 2: Main steps of the proposed prognostic method.

\subsection{Feature extraction and reduction}

This section presents the feature extraction and reduction technique. The features are first extracted from raw monitoring signals by using wavelet packet decomposition. This latter technique allows calculating the energy of the nodes at each level of decomposition. Then, a dimensionality reduction is performed on the extracted features by using the ISOMAP technique. ISOMAP is a manifold learning technique based on pairwise distances derived from high dimensional data [15] and is a way of enhancing classical multi-dimensional scaling (MDS). The purpose of the ISOMAP technique is to find a small number of features that represents a large number of observed dimensions. It has the advantage to be nonlinear, non-iterative and gives 
global optimal solutions [15].

Given a set of data points $S_{m}=\left\{x_{i}, i \in M=\{1,2, \ldots, m\}\right\} \subset R^{n}$, the geodesic distance is defined by the length of the shortest curve that connects two points on the manifold. The aim of ISOMAP is to find an embedding of the given dataset $S_{m}$ into a lower dimensional space $R^{d}$, where $d$ is the intrinsic dimension of the manifold. The ISOMAP algorithm is summarized by the following three steps.

1. Construction of the neighborhood graph over all observations by connecting the $i j-t h$ point if point $i$ is one of the $k$ nearest neighbors of $j$ (or if the distance between them is less than $\varepsilon$ ). Then, put the lengths of the edges equal to $d_{i j}$.

2. Approximation of the geodesic distances with the shortest paths between points in the graph.

3. Finding the d-dimensional embedding by applying classical MDS to the geodesic paths found in step 2. For this point, one firstly computes the matrix $k=-H S H / 2$, where $S$ is the matrix of the squared geodesic distances among the points and $H$ is a centering matrix for which the generic element is defined by $h_{i j}=\delta_{i j}-1 / m$, with $\delta_{i j}$ denoting the Kronecker's delta. Then, the first $d$ eigenvalues $\left\{\lambda_{1}, \lambda_{2}, \ldots, \lambda_{d}\right\}$ of the matrix $K$ and the corresponding eigenvectors $\left\{v_{1}, v_{2}, \ldots, v_{d}\right\}$ are taken. The $p^{\text {th }}$ of the $i^{\text {th }}$ point in the new space is then defined as $z_{i, p}=\sqrt{\lambda_{p}} v_{i, p}, p=1,2, \ldots, d$.

\subsection{Support vector regression}

The theory of support vector machine (SVM) has been introduced by Vapnik [22]. SVM is divided into two main categories: support vector classification (SVC) and support vector regression (SVR). SVR is the most common application form of SVMs. It has been proposed in 1997 by Vapnik et al. [23]. The main objective of SVR is to estimate a functional relation between input and output random variables under the assumption that the joint distribution $P$ of the input and output variables is completely unknown. The model created by SVR depends only on a subset of the training data, because the cost function for the model construction ignores all training data that are close within a threshold $\epsilon$ to the model prediction. Regression estimation can be formalized as the problem of inferring a function $y=f(x)$ based on a training set $X=\left\{\left(x_{i}, d_{i}\right), i=1, \ldots, l\right\}$, where $x_{i} \in R^{n}$ is the $i^{t h}$ 
input vector for the $i^{\text {th }}$ training example, $d_{i} \in R$ is the target value for the $i^{\text {th }}$ training example and $l$ is the number of training set. Training a support vector regression (SVR) machine is equivalent to finding a regression function of the form:

$$
f(x)=\sum_{i=1}^{l}\left(\alpha_{i}-\alpha_{i}^{*}\right) k\left(x_{i}, x\right)+b
$$

where $k\left(x_{i}, x\right)$ is a positive definite kernel function, $\alpha=\left(\alpha_{1}, \alpha_{2}, \ldots, \alpha_{l}\right)^{T}$, $\alpha^{*}=\left(\alpha *_{1}, \alpha *_{2}, \ldots, \alpha *_{l}\right)^{T}$ and $b$ the parameters of the model. To find $\alpha_{i}, \alpha_{i}^{*}, i=$ $1, \ldots, l$ one needs to minimize the objective function:

$$
\sum_{i, j=1}\left(\alpha_{i}-\alpha_{i}^{*}\right)\left(\alpha_{j}-\alpha_{j}^{*}\right) k\left(x_{i}, x_{j}\right)+\varepsilon \sum_{i=1}^{l}\left(\alpha_{i}+\alpha_{i}^{*}\right)-d \sum_{i=1}^{l}\left(\alpha_{i}-\alpha_{i}^{*}\right)
$$

which is subject to the following expression:

$$
\sum_{i=1}^{l}\left(\alpha_{i}-\alpha_{i}^{*}\right)=0 \text { and } \alpha_{i}, \alpha_{i}^{*} \in[0, C]
$$

where $\varepsilon$ and $C$ are the hyper-parameters.

The kernel $k\left(x_{i}, x\right)$ can have different forms; the most used one is the Gaussian function $k\left(x_{i}, x\right)=\exp \left(-\frac{\left\|x_{i}-x\right\|^{2}}{\sigma^{2}}\right)$, where $\sigma>0$ is the kernel's width.

The training of SVR needs to solve the quadratic optimization problems given in Eq. (2) and Eq. (3) with 2 parameters. If $\alpha_{i}-\alpha_{i}^{*} \neq 0$ the sample $x_{i}$ is called support vector, if $0<\left|\alpha_{i}-\alpha_{i}^{*}\right|<C, x_{i}$ is an unbounded support vector, if $\left|\alpha_{i}-\alpha_{i}^{*}\right|=C, x_{i}$ is a bounded support vector and finally if $\left|\alpha_{i}-\alpha_{i}^{*}\right|=0, x_{i}$ is a non support vector. With the notion of support vector, the regression function given in Eq. (1) of SVR can be simplified as:

$$
f(x)=\sum_{x_{i} \in S V}^{l}\left(\alpha_{i}-\alpha_{i}^{*}\right) k\left(x_{i}, x\right)+b
$$

\subsection{RUL estimation}

The diagram of the SVR method proposed for bearing's fault prognostics is given in figure 3. The method is decomposed into two main steps. The first step is done off-line and aims at generating an appropriate model that allows describing the evolution of the bearing's degradation. Specifically, the 


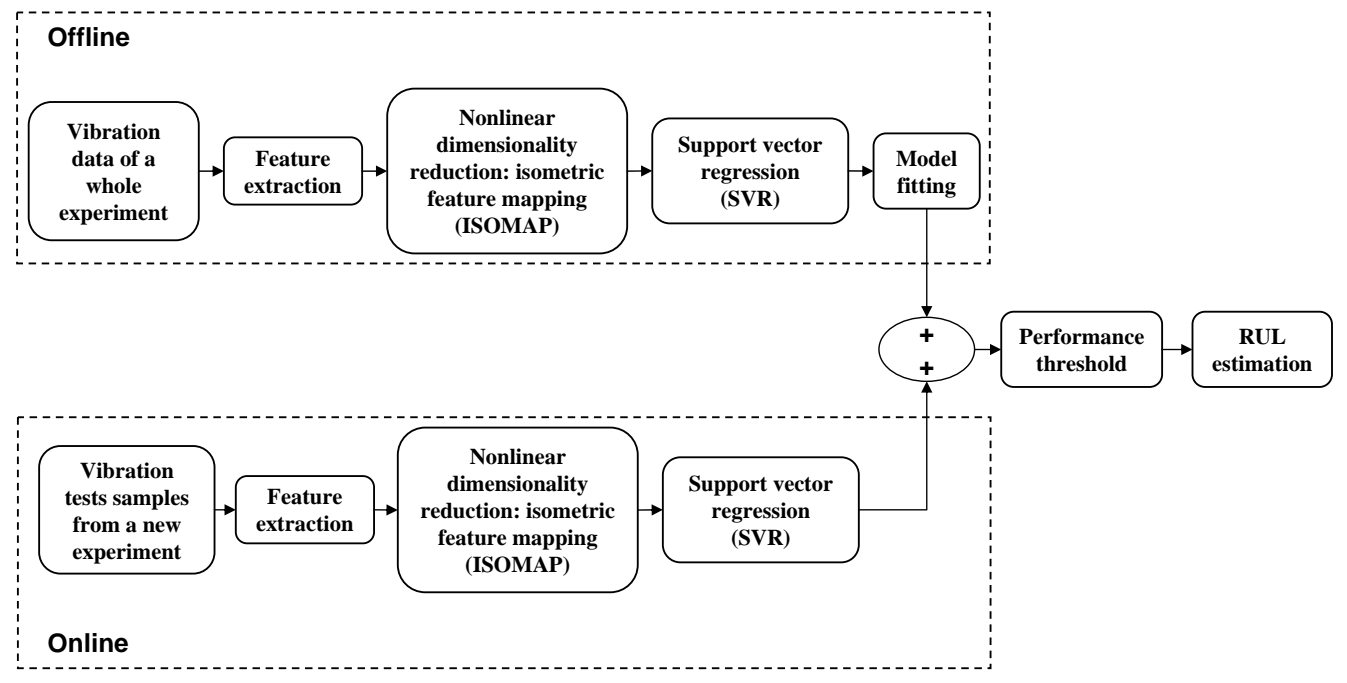

Figure 3: Framework of the RUL estimation procedure.

goal of this step is to build the regression model by using the SVR technique. The second step, which is achieved on-line, utilizes the model generated in the first step to continuously assess the health state of the bearing and to predict its future one leading to the calculation of its RUL.

\section{Application and results}

\subsection{Description of the experimental platform PRONOSTIA}

PRONOSTIA is an experimental platform dedicated to test, verify, and validate methods related to bearings' health assessment, diagnostics, and prognostics. A general overview of the platform is shown in figure 4. The main purpose of PRONOSTIA is to provide real data related to bearings' degradations. In this platform, the experiments are carried out by applying loads on the bearings exceeding the loads allowed by the catalog in order to accelerate their degradation. The bearings with the reference NSK 6804DD, which can operate at a maximum speed of $13000 \mathrm{rpm}$, and a load limit of $4000 \mathrm{~N}$ are used in this application.

PRONOSTIA is composed of two main parts: a first part related to the speed variation, and a second part dedicated to load profile generation. The speed variation part is composed of a synchronous motor, a shaft, a set of bearings, and a speed controller. The synchronous motor develops a power 


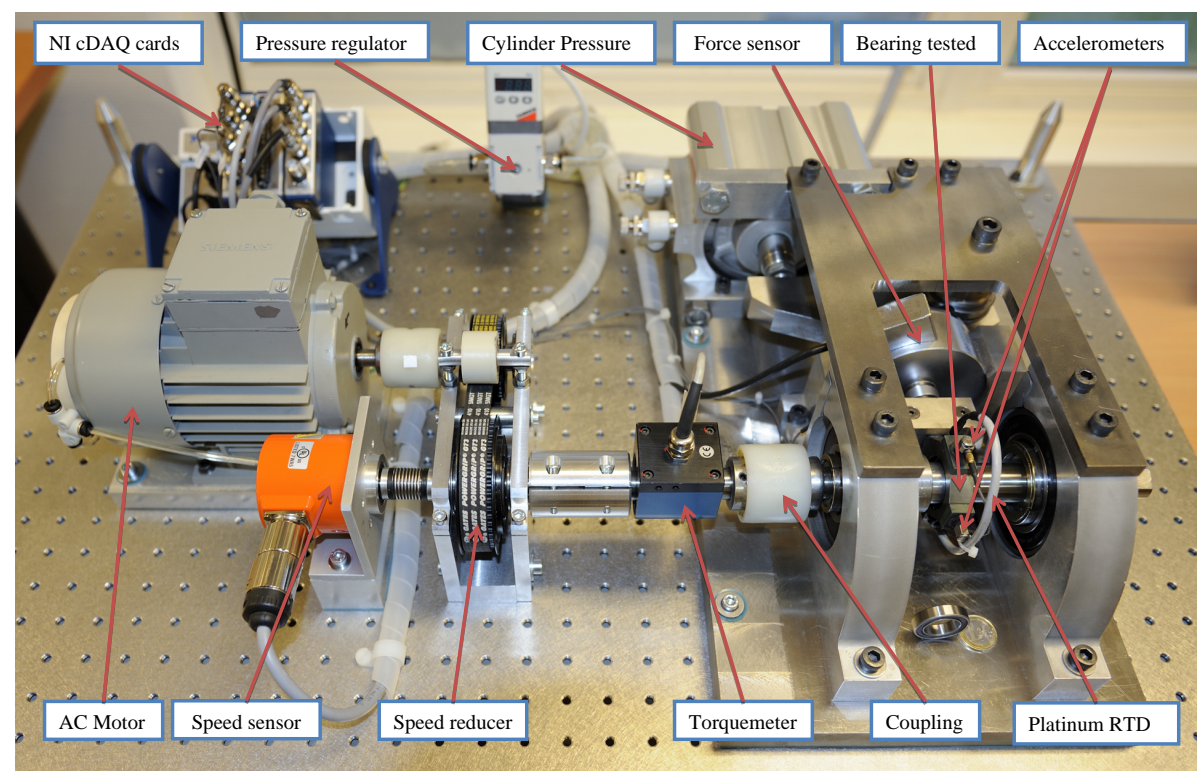

Figure 4: The experimental platform Pronostia.

equal to $1.2 \mathrm{~kW}$, and its operational speed varies between 0 and $6000 \mathrm{rpm}$. The load part is composed of a hydraulic jack connected to a lever arm used to create different loads to degrade the bearing mounted on the platform. The radial load can be varied between 0 and $10000 \mathrm{~N}$, and the operating speed of the bearing can be controlled within the interval 0 - $2000 \mathrm{rpm}$. The force delivered by the pneumatic jack is indirectly applied on the external ring of the bearing through a clamping ring (figure 5-(b)). The effort is transmitted by a lever arm in rotation, which applies the load on the clamping ring.

Two high frequency accelerometers (DYTRAN 3035B) are mounted, one horizontally and one vertically, on the housing of the tested roller bearing to pick up the horizontal and the vertical accelerations. In addition, the monitoring system includes one temperature probe to record the temperature of the tested bearing. A speed sensor and a torque sensor are also available on the PRONOSTIA platform. The sensors are connected to a data acquisition card (NI DAQCard-9174) to provide the user with monitoring data. The sampling frequency is set to $25600 \mathrm{~Hz}$, and the vibration data provided by the two accelerometers are collected every 1 second. 


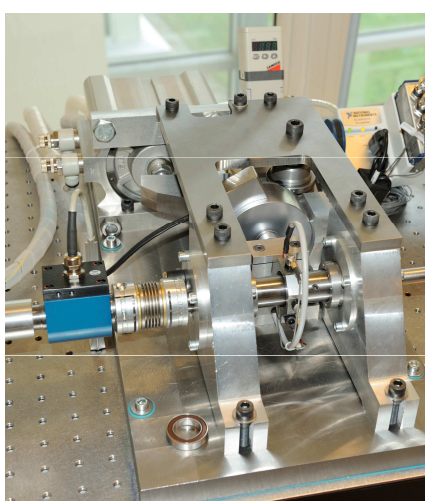

(a)

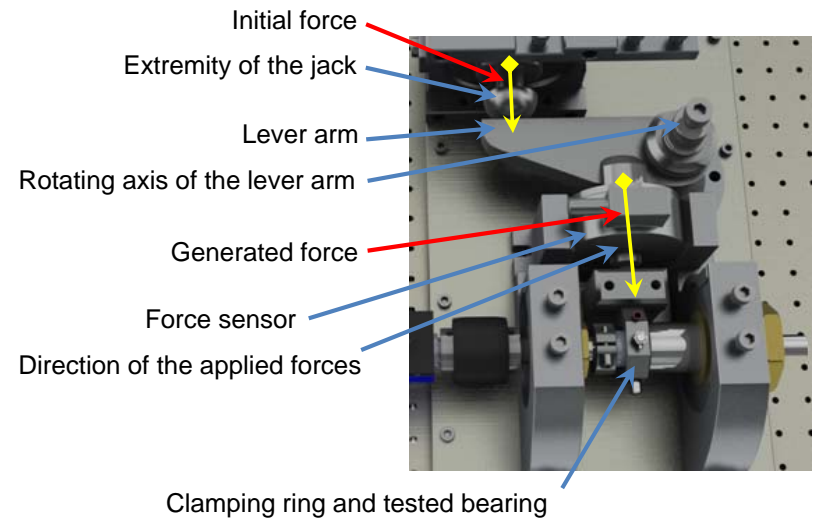

(b)

Figure 5: Multiplication system and load transmission: (a) top view and (b) elements of the transmission chain.

\subsection{Experimental results}

Three experimentations corresponding to three degraded bearings are used to test the method. The durations of the experiments are: 6 hours and 50 minutes, 6 hours and 16 minutes, and 4 hours and 30 minutes. Two vibration signals (vertical and horizontal accelerometers) collected from each experiment are used for feature extraction. More information about the tested bearings is given in table 1 . Moreover, the results presented in this paper are valid within the framework defined by the following assumptions.

1. The tested bearings have the same reference and are provided by the same manufacturer.

2. No faults are initiated on the bearings before the beginning of each experiment.

3. During the experiments, each bearing, which is initially new, is used until it is failed. Furthermore, the degradation of each bearing is supposed to be any (fault of the balls, the ring, the races or a combination of all these faults).

4. The data of each experiment are then used to extract features and learn the degradation model of the corresponding bearing. 
5. The bearings which are degraded under the same operating conditions (same load, same velocity, etc.) form a same group. The model learned for a group of bearings is then used to estimate the RUL of another bearing belonging to the same group.

\begin{tabular}{|c|c|c|c|}
\hline Duration of the tested bearings & $6 \mathrm{~h} 50$ & $6 \mathrm{~h} 16$ & $4 \mathrm{~h} 30$ \\
\hline Loading (N) & 4000 & 4000 & 5000 \\
\hline Speed(RPM) & 1800 & 1800 & 1500 \\
\hline Training error & 0.0494 & 0.0248 & 0.0187 \\
\hline
\end{tabular}

Table 1: Bearings dataset from PRONOSTIA test rig.

\subsubsection{Selection of the optimal parameters of ISOMAP and SVR}

The input parameters of ISOMAP technique are $k$ or $\epsilon$. Usually, these parameters are specified by the user. However, in this contribution the parameters are selected by using the optimization method proposed in [24]. The implementation of the method led to a value of $k$ equal to 4 (figure 6). After feature reduction, and before using the SVR for model construction,

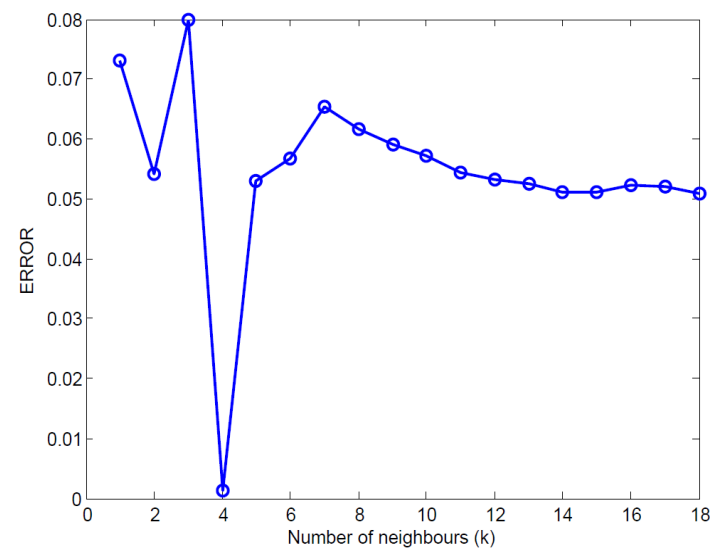

Figure 6: Optimized value of the ISOMAP parameter $k$.

it is necessary to select the optimal kernel that gives the minimum error of training. In the following tests, a Gaussian kernel with a width parameter $\sigma$ equal to $10^{-5}$ is considered. Indeed, the value of the kernel width can affect the number of support vectors used to construct the regression 


\begin{tabular}{|c|c|c|c|}
\hline Kernel & Gaussian $\sigma=10^{-5}$ & Polynomial & Radial Basis Function \\
\hline RMSE & 0.014 & 0.294 & 0.095 \\
\hline
\end{tabular}

Table 2: Performance of training data by different kernels.

function. In this work, the kernel width values were varied in the range of $\left\{10^{-6}, 10^{-5}, \ldots, 1\right\}$ and the optimal value is set $10^{-5}$.

\subsubsection{Feature extraction and reduction}

In this application, eight features are extracted from the vertical and horizontal accelerometers (vibration signals) of each degraded bearing by using the wavelet packet decomposition technique. The features represent the percentage of energy at each level of decomposition on the raw vibration signals. An example of three features plotted together is shown in figure 7 .

The eight features extracted for each bearing are then reduced to one

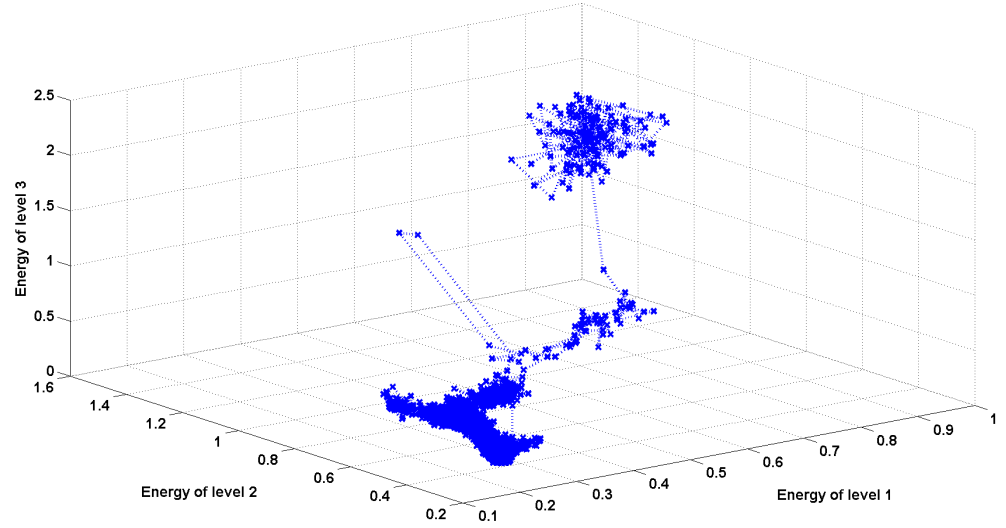

Figure 7: Three features extracted from the bearing degraded within 6 hours and 50 minutes.

variable (called health indicator) by using the ISOMAP technique. Figure 8 shows the eight features extracted from the vibration signals of the bearing degraded within 6 hours and 50 minutes, and figure 9 presents the health indicator obtained after reducing the eight features by using the ISOMAP technique. 




Figure 8: Eight features extracted from the bearing degraded within 6 hours and 50 minutes.

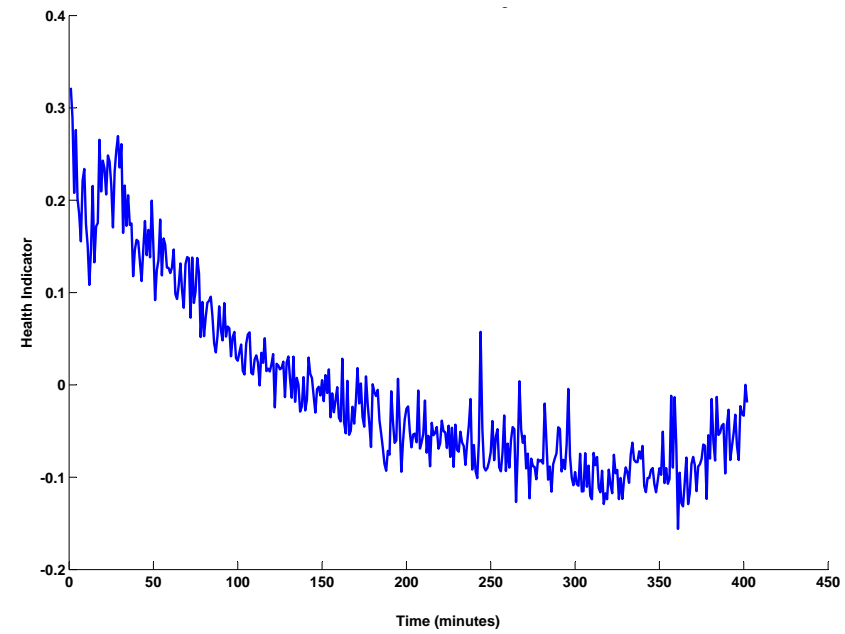

Figure 9: The health indicator extracted from the eight features by using the ISOMAP technique.

\subsubsection{SVR models and RUL estimation}

The support vectors obtained by using the SVR, and the predicted models corresponding to the three experiments are shown in figures 10, 11 and 




Figure 10: Degradation evolution obtained from SVR for the experiment corresponding to 4 h30.



Figure 11: Degradation evolution obtained from SVR for the experiment corresponding to $6 \mathrm{~h} 16$.

12. The support vectors are then fitted to exponential regressions used to predict the degradation level of each bearing and calculate the correspond- 




Figure 12: Degradation evolution obtained from SVR for the experiment corresponding to $6 \mathrm{~h} 50$.

ing RUL values. Moreover, to calculate the confidence value of each RUL, upper and lower bounds are added to the regressions as shown in figure 13. The calculation of the RUL is done on the regression models by defining a failure threshold for each degradation. The failure threshold can be given by experts, learned automatically from experience data, defined according to performance criteria, etc. In this application, the threshold is set equal to 1.4 of the root mean square of the vibration signal. This value corresponds to the accepted level of degradation in the tested bearings.

The RUL and the associated confidence at $95 \%$ are calculated by using the following equation:

$$
R U L_{u / l}\left(t_{j}\right)=\left(t^{\text {failure }}-t_{j}\right) \pm\left(t^{\text {failure }}-t_{95}^{\text {failure }}\right)
$$

\begin{tabular}{|c|c|c|c|c|}
\hline Test & size data & SSE & R-square & RMSE \\
\hline Test1 $(6 h 50)$ & 24632 & 0.3339 & 0.9695 & 0.02158 \\
\hline Test1 $(6 h 16)$ & 22589 & 0.3279 & 0.9645 & 0.02245 \\
\hline Test1 (4h30) & 16366 & 0.3268 & 0.9693 & 0.02316 \\
\hline
\end{tabular}

Table 3: Fitting data. 


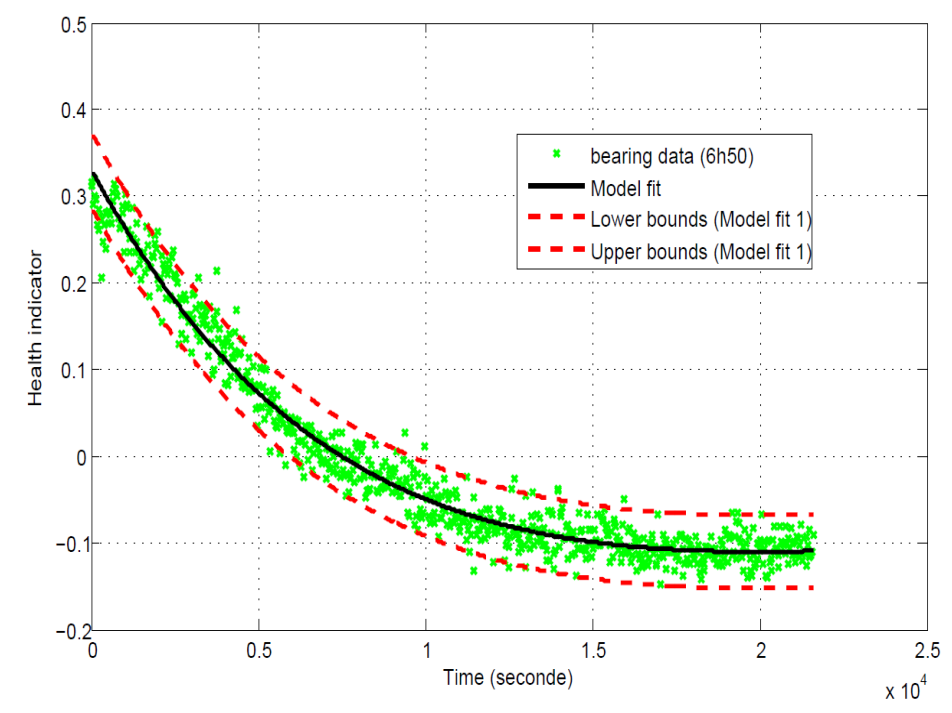

Figure 13: fitting data after reduction.

The time $t_{j}$ is obtained by inverting the model $y(t)=a_{1} \cdot e^{b_{1} \cdot t}+c_{1}$ :

$$
t_{j}=\frac{1}{b_{1}} \log \left(\frac{y-c_{1}}{a_{1}}\right)
$$

The RUL corresponding to the experiment which duration is 4 hours and 30 minutes is given in figure 14. From the figure it can be seen that the RUL in the middle of predictions is under the real RUL value. This can be seen as a pessimistic prediction, which is suitable for maintenance planing (do the maintenance interventions before the real time of a failure).

\section{Conclusion and future work}

This paper presented a prognostic method based on a nonlinear feature reduction (ISOMAP) and SVR. The method belongs to data-driven prognostics and the application was on bearings' degradations. Moreover, the method can be applied on degradation of other critical components (batteries, train doors, gearboxes, etc.) at a condition that appropriate sensors are available. For the ISOMAP technique, the input parameters were defined by using an optimization approach. In the case of SVR, a Gaussian kernel was considered. Then, exponential regression models were used to fit the support 




Figure 14: Predicted RUL of a bearing degraded in 4 hours and 30 minutes.

vectors obtained from SVR and the derived models allowed to calculate the RUL of the degraded bearings.

The proposed contribution concerned critical components operating under constant conditions (same speed, load, temperature, etc.) and without any maintenance intervention during the degradation. Indeed, the performance of the component is only deceasing in time. These two aspects (variable operating conditions and maintenance interventions) are the ongoing works which may help generalizing the method.

\section{References}

[1] K. Medjaher, D. A. Tobon-Mejia, N. Zerhouni, Remaining useful life estimation of critical components with application to bearings, IEEE Transactions on Reliability 61 (2) (2012) $292-302$.

[2] A. K. Jardine, D. Lin, D. Banjevic, A review on machinery diagnostics and prognostics implementing condition-based maintenance, Mechanical Systems and Signal Processing 20 (7) (2006) 1483 - 1510.

[3] A. Heng, S. Zhang, A. C. C. Tan, J. Mathew, Rotating machinery prognostics: state of the art, challenges and opportunities, Mechanical Systems and Signal Processing 23 (3) (2009) $724-739$. 
[4] Y. Shao, K. Nezu, Prognosis of remaining bearing life using neural networks, Journal of Systems and Control Engineering part I, 214(3) (2000) $217-230$.

[5] L. Haitao, Z. Wenbiao, G. Huairui, Predicting remaining useful life of an individual unit using proportional hazards model and logistic regression model, in: Reliability and Maintainability Symposium, RAMS'06, 2006, pp. $127-132$.

[6] N. Gebraeel, M. Lawley, R. Liu, V. Parmeshwaran, Residual life predictions from vibration-based degradation signals: a neural network approach, IEEE Transactions on Industrial Electronics 51 (2004) 694-700.

[7] R. Huang, L. Xi, X. Li, C. Liu, H. Qiu, J. Lee, Residual life predictions for ball bearings based on self-organizing map and back propagation neural network methods, Mechanical Systems and Signal Processing 21 (1) (2007) 193-207.

[8] J. H. Yan, J. Lee, Degradation assessment and fault modes classification using logistic regression, Journal of Manufacturing Science and Engineering Transactions of the ASME 127 (4) (2005) 912-914.

[9] L. Lingjun, H. Jie, H. Wei, D. Xinmin, H. Zhengjia, Condition evaluation for mechanical equipment by means of support vector data description, Chinese Journal of Mechanical Science and Technology 24 (12) (2005) 1426-1429.

[10] F. Xi, Q. Sun, G. Krishnappa, Bearing diagnostics based on pattern recognition of statistical parameters, Journal of Vibration Control 6 (2000) 75-92.

[11] L. J. P. V. D. Maaten, E. O. Postma, H. J. V. D. Herik, Dimensionality reduction: A comparative review, Tilburg University Technical Report, tiCC-TR 2009-005 (2009).

[12] J. E. Jackson, A user's guide to principal components, Wiley, New York, 1991.

[13] L. Liao, J. Lee, A novel method for machine performance degradation assessment based on fixed cycle features test, Journal of Sound Vibration 326 (2009) 894-908. 
[14] A. Malhi, R. Gao, PCA-based feature selection scheme for machine defect classification, IEEE Transactions on Instrumentation and Measurement 53 (2004) 1517-1525.

[15] J. Tenenbaum, V. De Silva, J. C. Langford, A global geometric framework for nonlinear dimensionality reduction, Science 290 (2000) 2319 2323 .

[16] B. Schölkopf, A. Smola, Learning with kernels, MIT Press, 2002.

[17] R. Collobert, S. Bengio, SVMTorch: Support vector machines for largescale regression problems, Journal of Machine Learning Research 1 (1) (2001) $143-160$.

[18] A. Demiriz, K. Bennett, C. Breneman, M. Embrechts, Support vector machine regression in chemometrics, Computing Science and Statistics, 2001.

[19] M. Lebold, M. Thurston, Open standards for condition-based maintenance and prognostic systems, in: Proc. 5th Maintenance and Reliability Conference (MARCON), 2001.

[20] AFNOR, Condition monitoring and diagnostics of machines - prognostics - part 1: General guidelines. NF ISO 13381-1 (2005).

[21] D. A. Tobon-Mejia, K. Medjaher, N. Zerhouni, CNC machine tool's wear diagnostic and prognostic by using dynamic bayesian networks, Mechanical Systems and Signal Processing 28 (2012) 167 - 182.

[22] V. Vapnik, The Nature of Statistical Learning Theory, Springer, New York, 1995.

[23] V. Vapnik, S. Golowich, A. Smola, Support vector method for function approximation, regression estimation, and signal processing, Advances in Neural Information Processing Systems 9 (1997) 281-287.

[24] O. Samko, A. Marshall, P. Rosin, Selection of the optimal parameter value for the ISOMAP algorithm, Pattern Recognition Letters 27 (9) (2006) 968 - 979. 